INRA Prod. Anim., 2007, 20 (1), 93-100

\section{Impact des conditions de pré-abattage sur le stress et le bien-être des animaux d'élevage}

E.M.C. TERLOUW', C. ARNOULD'², B. AUPERIN ${ }^{3}$, C. BERRI ${ }^{4}$, E. LE BIHAN-DUVAL 4 , F. LEFEVRE ${ }^{3}$, J. LENSINK 5 , L. MOUNIER ${ }^{6-7}$

${ }^{1}$ INRA, UR370 Qualité des Produits Animaux, F-63122 Saint-Genès Champanelle, France

2 INRA, CNRS, Université de Tours, Haras Nationaux, UMR85 Physiologie de la Reproduction et des Comportements, F-37380 Nouzilly, France

${ }^{3}$ INRA, UR1037 Station Commune de Recherches en Ichtyophysiologie, Biodiversité et Environnement, F-35042 Rennes, France

4 INRA, UR83 Recherches Avicoles, F-37380 Nouzilly, France

5 Institut Supérieur d'Agriculture, 48 boulevard Vauban, F-59046 Lille, France ${ }^{6}$ INRA, UR1213 Herbivores, F-63122, Saint-Génès Champanelle, France

${ }^{7}$ Ecole Nationale Vétérinaire de Lyon, 1 avenue Bourgelat, F-69280 Marcy l'Etoile, France Courriel :Claudia.Terlouw@clermont.inra.fr
Les procédés d'abattage générateurs de stress ${ }^{1}$ varient selon l'espèce considérée. Un état de stress chez l'animal peut être d'origine physique : fatigue, privation de nourriture, mauvaises conditions thermiques ou qualité d'air ou d'eau, douleur due aux agressions, heurts, ou coups. Il peut aussi avoir une origine psychologique : perturbations sociales (séparation sociale, mélanges d'animaux non familiers, densité non adaptée), peur (environnements non familiers, présence de l'homme). Sauf dérogation, l'étourdissement (réversible ou non) est obligatoire avant la saignée. La méthode choisie dépend de l'espèce. L'étourdissement électrique ou électronarcose est induit par le passage d'un courant à travers le cerveau produisant une décharge des neurones qui ne fonctionnement plus jusqu'à leur repolarisation. L'introduction d'un poinçon dans le cerveau détruit une partie du cerveau, et provoque une perte de conscience qui est irréversible si le poinçon est correctement positionné (Daly et al 1987). L'étourdissement au gaz est pratiqué par immersion de l'animal dans un mélange gazeux souvent contenant du $\mathrm{CO}_{2}$, de l'argon et/ou de l'azote (Deiss et al 2006). D'autres techniques existent pour le poisson. Quelle que soit la méthode d'étourdissement utilisée, l'opération est souvent associée à un inconfort physique et psychologique. Dans tous les cas, la bonne application de l'étourdis- sement nécessite une bonne formation de l'opérateur, et un bon entretien du matériel (Grandin 2005). Contrairement aux autres espèces, certaines espèces de poissons ne sont pas saignées après l'étourdissement ou la mise à mort.

A l'abattage, des mesures comportementales, physiologiques et métaboliques sont utilisées pour apprécier le niveau de stress et de bien-être ${ }^{1}$. Les mesures physiologiques et métaboliques sont influencées à la fois par l'activité physique et par le stress psychologique, lui-même pouvant provoquer des réponses motrices. La fréquence cardiaque et les taux de cortisol/corticostérone et d'adrénaline et noradrénaline sont souvent interprétés en terme d'activité physique et/ou de réponse de stress. Le rapport hétérophile/lymphocyte est utilisé classiquement comme indicateur d'une réponse de stress. L'établissement d'un électroencéphalogramme après un stimulus visuel, auditif ou somesthésique permet de mesurer la perte efficace de conscience (Robb et al 2000, Martoft et al 2002, Raj 2004). L'augmentation des taux sanguins de lactate ou de créatine kinase reflète en général une activité physique accrue, qui peut être associée à un état de stress. Les mesures comportementales, comme le comportement de repos, l'activité physique forcée (liée aux manipulations), les tentatives de fuites, les chutes, le niveau d'agression sont des indicateurs de l'état de stress de l'animal et, de plus, facilitent l'interprétation des mesures physiologiques. Certains tests comportementaux permettent d'évaluer l'état émotionnel de l'animal. Chez l'oiseau par exemple, le niveau de peur s'évalue à travers la durée d'immobilité tonique provoquée expérimentalement par une contention. Des tests de réactivité à des situations d'aversion effectués avant l'abattage peuvent parfois aider à prédire ou mieux interpréter les réactions de l'animal à certains procédés de l'abattage.

Les mesures du métabolisme musculaire post mortem, y compris l'évolution de la température et du $\mathrm{pH}$, permettent d'apprécier le niveau d'activité physique, et parfois de stress, de l'animal avant son abattage. Ainsi, un $\mathrm{pH}$ à $24 \mathrm{~h}$ élevé reflète une déplétion des réserves glycolytiques musculaires, suggérant des efforts importants ante mortem (Debut et al 2003), parfois associés à des taux d'adrénaline élevés (Lensink et al 2001a). Une température musculaire élevée et/ou un $\mathrm{pH}$ bas dans les minutes ou heures qui suivent l'abattage sont indicateurs d'activité et/ou de stress élevé dans les minutes qui précèdent l'abattage (Terlouw et Rybarczyk 2007). Chez le poisson, le stress à l'abattage peut conduire à une diminution rapide de $\mathrm{pH}$ et une entrée

${ }^{1}$ Les termes «stress» et «bien-être» dans ce texte sont utilisés comme définis dans l'article introductif de ce numéro spécial de INRA Productions Animales (Veissier et al 2007). Ainsi, le stress fait référence à l'état émotionnel, comportemental et physiologique d'un animal qui se sent menacé. Le bien-être d'un animal stressé est diminué. 
en rigor mortis accélérée (Morzel et al 2003). Toutefois, quand le stress entrâ̂ne une diminution de l'activité physique avant l'abattage, l'évolution du $\mathrm{pH}$ peut être normale.

\section{1 / Volailles}

Les volailles sont élevées soit au sol en grands groupes de plusieurs milliers d'individus (cas des volailles de chair), soit en cage de quelques individus (cas des poules pondeuses). Avant leur ramassage dans les bâtiments d'élevage, les animaux sont mis à jeun (au moins $8 \mathrm{~h}$ avant l'abattage). Ils sont ensuite mis en caisse et chargés dans un camion pour le transport (durée ne pouvant excéder $2 \mathrm{~h}$ pour les poulets de type Label et au maximum $8 \mathrm{~h}$ pour les autres types de productions). A l'arrivée des camions, les caisses sont généralement déchargées et les animaux mis en attente. Les caisses sont ensuite chargées sur un tapis roulant qui les conduit vers le poste d'accrochage. Les oiseaux sont suspendus manuellement par leurs pattes sur la chaîne d'abattage et transportés jusqu'au bac d'électronarcose (principal mode d'étourdissement utilisé en France) où la tête des oiseaux est plongée dans un bain d'eau électrifié. Les oiseaux sont saignés dès la sortie du bac.

La mise à jeun des oiseaux avant leur abattage peut avoir des conséquences en terme de bien-être. On observe après $2 \mathrm{~h}$ de jeûne une augmentation du taux de corticostérone, traduisant une adaptation métabolique ou un stress psychologique liée au retrait de l'aliment (Kannan et Mench 1996). Après 24 h, il peut $y$ avoir une perte importante de poids, jusqu'à $10 \%$ du poids vif (Warriss et al 1999). Le jeûne affecte aussi les réserves en glycogène du foie et du muscle, ce qui pourrait rendre les oiseaux plus sensibles aux stress subis pendant le transport et à l'abattoir (Kotula et Wang 1994).

Le ramassage est sans doute à l'origine des plus gros problèmes de bien-être rencontrés. Les oiseaux sont ramassés par les pattes et transportés la tête en bas jusqu'aux caisses de transport. Cette manipulation est à l'origine de fractures et d'hématomes au niveau des pattes et des ailes (Gregory et Wilkins 1989). Chez le poulet, le maintien des animaux la tête en bas et la mise en caisse provoquent une élévation du taux de corticostérone (Kannan et Mench 1996). Cependant, le temps passé en caisse n'affecte pas cette réponse, probablement parce que cette élévation est déjà maximale suite aux manipulations précédant la mise en caisse (Kannan et al 1997b). Des dispositifs de ramassage automatique des poulets existent, mais ils restent encore peu utilisés. Pourtant, les poulets ramassés avec de telles machines ont des réponses de peur, évaluées par un test d'immobilité tonique, des taux de corticostérone et des taux de blessures plus faibles que ceux ramassés manuellement (Duncan et al 1986, Knierim et Gocke 2003).

Le transport regroupe plusieurs situations qui peuvent affecter le bien-être des oiseaux : un changement d'environnement, des mouvements du camion à l'origine de traumatismes physiques, un environnement climatique défavorable (température/humidité, courants d'air). Ainsi, la durée du transport et le temps d'attente avant celui-ci augmentent le niveau de peur estimé par la durée d'immobilité tonique (Cashman et al 1989). Le nombre d'individus trouvés morts à l'arrivée du camion augmente avec la durée de transport et en cas de température élevée (Warriss et al 1992). La réponse de stress évaluée par les taux de corticostérone et le rapport hétérophiles/lymphocytes augmente également avec la durée du transport (Mitchell et al 1992). La diminution des réserves en glycogène hépatique et musculaire (Warriss et al 1999) et l'augmentation des taux plasmatiques de créatine kinase s'expliquent par les efforts musculaires fournis. Les taux élevés de créatine kinase peuvent également être liés à une température ambiante élevée (Mitchell et al 1992).

A l'abattoir, la durée et les conditions d'attente sur les quais ainsi que les procédés liés à l'étourdissement peuvent affecter le bien-être des oiseaux. Ainsi, une élévation de corticostérone est observée chez les animaux soumis à de fortes chaleurs (Debut et al 2005). De même, l'accrochage avant l'électronarcose met clairement en cause le bienêtre des oiseaux. Il provoque une élévation des taux de corticostérone, même s'il n'a pas d'effet sur la durée d'immobilité tonique (Kannan et al 1997a, Debut et al 2005). Par ailleurs, l'accrochage déclenche des vocalisations et des battements d'ailes qui peuvent être assimilés à des tentatives de fuite (Gregory et Bell 1987, Debut et al 2005). Suite à des observations anatomiques, l'accrochage est considéré comme source de douleur physique forte (Gentle et Tilson, 2000). Chez les poules pondeuses élevées en cage, il provoque des fractures (Gregory et Wilkins 1989). L'étourdissement en atmosphère modifiée (gaz) qui limite la manipulation des oiseaux et évite l'accrochage avant l'étourdissement est de plus en plus préconisé mais encore peu développé en France. Toutefois, les conséquences en terme de souffrance dépendent des mélanges gazeux qui doivent être optimisés (Raj 2004, Lambooij et al 1999).

\section{2 / Pores}

Les porcs sont généralement élevés par groupes de 10 à 12 . Avant l'abattage, les porcs de différentes cases sont souvent regroupés sur le quai d'embarquement de l'éleveur, où la durée d'attente peut atteindre plusieurs heures. A l'arrivée du transporteur, ils sont chargés (normes : $235 \mathrm{~kg} / \mathrm{m}^{2}$ pour des porcs de $100 \mathrm{~kg}$ ) et partent directement à l'abattoir, ou alors ils font un ou plusieurs arrêts dans d'autres fermes pour compléter le chargement (normes : durée maximale de $12 \mathrm{~h}$ ). A l'abattoir, ils sont introduits dans des cases d'attente contenant entre 15 et parfois jusqu'à une cinquantaine de porcs selon l'abattoir. Selon les pratiques et l'heure d'arrivée, ils peuvent être abattus rapidement ou attendre 3 à 18 heures environ. En général, ils sont privés de nourriture 12 à $24 \mathrm{~h}$ avant l'abattage. Les porcs de différentes fermes ne sont pas mélangés. Avant la saignée, les porcs sont conduits au poste d'étourdissement pour subir une anesthésie électrique ou gazeuse.

Un équipement bien conçu pour le chargement dans le camion évite les glissades et favorise une progression fluide des porcs vers le camion, limitant le stress. Le chargement, comme le déchargement, nécessite dans tous les cas un effort physique et provoque une augmentation de la fréquence car-

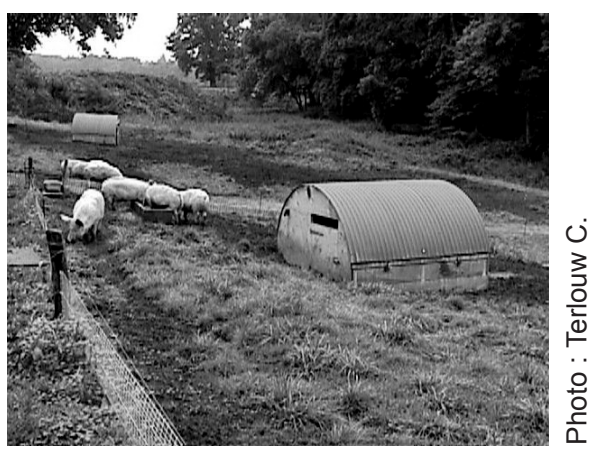

Photo 1 : Les porcs élevés à l'extérieur sont moins agressifs pendant le mélange avant l'abattage. 
Figure 1. Fréquence cardiaque de porcs (" : orcs Large White, : porcs Duroc) au repos dans la loge, puis pendant les différentes étapes du transport (Terlouw et Rybarczyk 2007).

\section{Battements par minute}

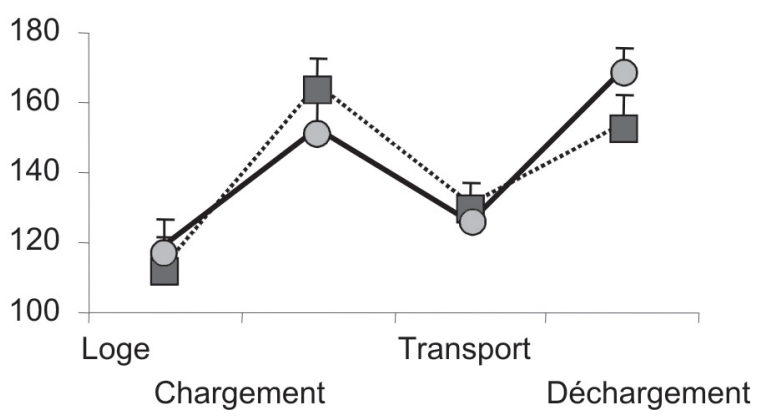

diaque et des taux sanguins de cortisol et de créatine kinase (Brown et al 2005, Kim et al 2004, figure 1). Pendant le transport la limitation de changements abrupts de vitesse et de direction, et une température ambiante adaptée, permettent aux porcs de se stabiliser et même de se reposer. Dans ce cas, la durée n'influence pas les réponses physiologiques ou métaboliques (Hambrecht et al 2005). Ainsi, si l'équipement est bon, le transport ne provoque pas nécessairement un inconfort important.

Les effets de la durée d'attente à l'abattoir dépendent également des conditions. Le mélange des porcs est souvent le facteur le plus stressant. Les agressions entre porcs donnent lieu à des augmentations des taux sanguins de cortisol, d'adrénaline et de métabolites et le $\mathrm{pH}$ ultime des viandes est plus élevé (Fernandez et al 1994, Terlouw et al 2006). Même si jusqu'à $12 \mathrm{~h}$ de privation de nourriture, il n'y a pas d'inconfort physique prononcé (Beattie et al 2002), le jeûne accentue les réponses aux combats (Fernandez et al 1994). Alors que nous ne disposons pas d'indicateur objectif du niveau de stress psychologique provoqué, les rencontres agressives sont cause de meurtrissures et de lésions cutanées parfois sévères qui provoquent de la douleur. Toutefois, si les groupes sont petits et si les porcs n'ont pas jeûné longtemps, l'attente peut être assimilée à un repos (Pérez et al 2002). L'agressivité est moindre chez des porcs élevés à l'extérieur (Terlouw et al 2006).

Au cours du chargement et déchargement et pendant la conduite au poste d'étourdissement les porcs sont manipulés par l'homme. La réactivité à l'homme est un facteur important dans les réponses au stress de l'abattage. Les porcs Large White s'approchant plus facilement de l'Homme dans des tests

effectués au cours de l'élevage, ont des muscles moins chauds après leur abattage, signe d'un niveau de stress et/ou d'une activité physique moindre juste avant l'abattage (Terlouw et Rybarzcyk 2007). Dans d'autres expériences, le contraire a été observé, probablement parce ce que les porcs moins peureux de l'homme étaient en queue de file et recevaient le plus de coups (Hemsworth et al 2002). Pendant la période de pré-abattage, les porcs sont également confrontés à des situations et environnements nouveaux. Cette nouveauté peut contribuer à la réactivité aux procédés d'abattage, car ceux qui exploraient plus souvent un objet non familier pendant des tests au cours de l'élevage avaient des $\mathrm{pH}$ ultimes plus élevés, signe de stress et/ou d'activité physique accrue pendant la période de pré-abattage (Terlouw et Rybarzcyk 2007).

Les différentes méthodes d'étourdissement présentent différents avantages l'abattage. et désavantages. L'intérêt du système d'anesthésie électrique, méthode principale en France, réside dans sa brièveté d'application. L'utilisation de l'anesthésie électrique nécessite la séparation des porcs au plus tard à l'entrée du restrainer, deux tapis roulants légèrement inclinés ou un seul tapis central qui portent le porc jusqu'aux électrodes. La séparation en file indienne contrarie l'instinct grégaire des porcs. Souvent, ils se chevauchent et se griffent dans les couloirs étroits. A cause de la position parfois incorrecte des électrodes, la quantité de courant nécessaire pour rendre l'animal insensible n'est pas toujours appliquée et le passage du courant peut alors être insuffisant et douloureux (Wotton et O'Callaghan 2002).

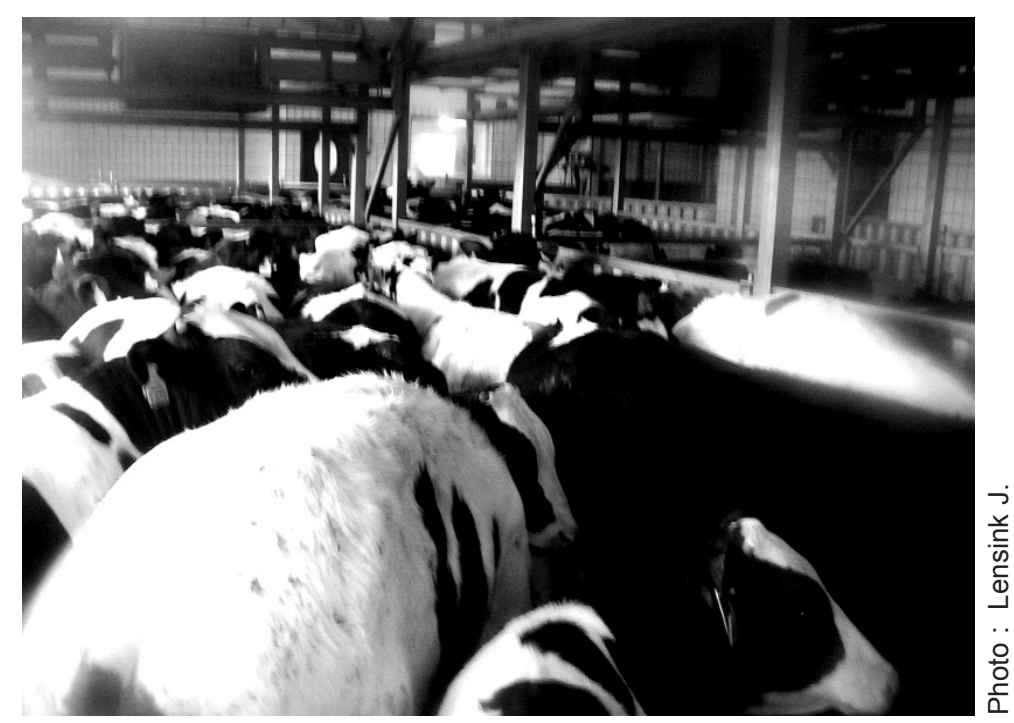

Photo 2 : A l'abattoir, les veaux de boucherie sont hébergés en groupe jusqu'à
Le système de narcose au $\mathrm{CO}_{2}$, un puit où les porcs sont descendus dans une nacelle, soit individuellement, soit en groupe, est beaucoup utilisé dans les pays nordiques. Chez l'homme, l'inhalation de $\mathrm{CO}_{2}$ peut induire une sensation asphyxiante et irrite les muqueuses (Gregory et al 1990). Chez le porc, on observe une excitation comportementale et des signes de souffrance respiratoire pendant la phase d'induction (Deiss et al 2006). Il reste à déterminer si le porc perd conscience avant ou après les signes de détresse (Hoenderken et al 1979, Martoft et al 2002).

\section{3 / Veaux de boucherie et bovins adultes}

L'enlèvement des bovins dans l'élevage se fait soit par groupe (veaux de 
boucherie, taurillons), soit individuellement (vaches de réforme ou parfois taurillons). Les bovins adultes de différents élevages peuvent être chargés dans le camion au fur et à mesure de la tournée et ainsi mélangés dans un même compartiment. Selon les normes européennes, la durée du trajet ne doit pas dépasser $8 \mathrm{~h}$, ou alors respecter des réglementations supplémentaires d'alimentation et d'abreuvement. L'abattage des bovins se fait soit en flux tendu après le déchargement, soit après une attente de 12 à $24 \mathrm{~h}$ en bouverie, le plus souvent en stalle individuelle. Les veaux de boucherie, en nombre plus important par élevage, partent habituellement directement de la ferme à l'abattoir. Leur transport dure généralement entre 1 et $4 \mathrm{~h}$. L'attente à l'abattoir se fait dans des loges communes contenant 20 à 40 veaux. Elle est relativement courte, entre $30 \mathrm{mn}$ et $4 \mathrm{~h}$, pour éviter les meurtrissures résultant du couchage des veaux fatigués. Les normes de densité pendant le transport dépendent du poids de l'animal, allant de $0,4 \mathrm{~m}^{2}$ pour un veau de $100 \mathrm{~kg}$, jusqu'à $2 \mathrm{~m}^{2}$ pour un adulte de $1000 \mathrm{~kg}$. En France, l'étourdissement des gros bovins et des veaux avant la saignée se fait à l'aide d'un pistolet à mèche captive. Pour les veaux, on peut également utiliser l'électronarcose (Lambooij et Spanjaard 1982).

De toutes les étapes du transport, le chargement semble le plus traumatisant. Les animaux sont sortis de leur case, manipulés par l'homme, puis rencontrent la situation non familière du transport. L'ensemble provoque une élévation de la cortisolémie, et une forte accélération de l'activité cardiaque (tableau 1) chez le veau, par rapport au niveau dans l'élevage (Lensink et al 2001b, Van de Water et al 2003). Le mode d'élevage, le type et la fréquence des contacts avec l'éleveur ont un effet sur les réactions de peur des animaux face à des situations nouvelles (Mounier et al 2007) et peuvent

Tableau 1. Effet du chargement, transport de $30 \mathrm{mn}$ et déchargement sur les réactions physiologiques de veaux (Lensink et al 2001b).

\begin{tabular}{|l|c|c|}
\hline & $\begin{array}{c}\text { Fréquence } \\
\text { cardiaque } \\
\text { (bpm) }\end{array}$ & $\begin{array}{c}\text { Cortisol } \\
\text { (ng/ml) }\end{array}$ \\
\hline Avant chargement & 110 & 5 \\
\hline Lors du chargement $^{2}$ & 190 & - \\
\hline Pendant le transport $^{2}$ & 135 & 16 \\
\hline Lors du déchargement $^{3}$ & 185 & - \\
\hline Après déchargement $^{3}$ & 120 & 5 \\
\hline
\end{tabular}

1 Moyennes de 32 veaux logés en paires.

2 Déterminé 5 minutes après la fin du transport.

3 Mesures effectuées $2 \mathrm{~h}$ après le déchargement et le retour des veaux dans leur groupe. dant la période du pré-abattage. L'élévation de la fréquence cardiaque peut donc être le résultat de l'effort physique de l'animal lié au chargement, mais également du stress psychologique. Par exemple, les veaux ayant reçu des contacts positifs et plus fréquents de leur éleveur étaient plus faciles à manipuler et avaient une fréquence cardiaque moins élevée que ceux qui avaient reçu des contacts plutôt brusques (Lensink et al 2001a, 2001b).

Le mélange d'animaux non familiers observé parfois lors de transferts des gros bovins provoque des interactions agressives. Chez les taurillons, on observe en plus une augmentation importante des chevauchements provoquant une fatigue musculaire (Kenny et Tarrant 1987). Le mélange des bovins a donc des conséquences négatives sur le bien-être animal et des répercussions négatives sur les qualités des viandes. Aujourd'hui, la hauteur des camions est limitée afin d'empêcher les chevauchements et l'attente des bovins adultes en bouverie se fait uniquement en stalles individuelles. Le mieux est de maintenir le groupe social. Les bovins y compris les veaux tendent à suivre leurs congénères. De plus, des taurillons maintenus dans leur groupe social avaient une moindre élévation de la cortisolémie au moment de la saignée et l'effet calmant du groupe était plus marqué si les animaux étaient familiers entre eux (Mounier et al 2006b). Le maintien du groupe facilite donc le chargement, limite le stress dû à la séparation et réduit les réactions émotionnelles des animaux au cours du transfert (Mounier et al 2006a).

Les conditions de chargement, principalement liées aux structures de l'élevage (couloir de contention, quai de chargement), déterminent en grande partie le niveau de stress psychologique et l'effort physique des bovins (Mounier et al 2006a). La présence de donc s'exprimer particulièrement pen- couloirs glissants, d'angles sur le trajet, de structures métalliques potentiellement blessantes peut provoquer non seulement des blessures, mais également induire un état de stress d'origine psychologique (Lensink et al 2001a, Van de Water et al 2003).

Le transport proprement dit provoque des réponses moins prononcées que le chargement. Chez le veau, on observe par rapport au chargement un ralentissement de l'activité cardiaque qui reste toutefois, ainsi que les taux plasmatiques de cortisol, de créatine kinase et de lactate, plus élevée que dans la loge (Sartorelli et al 1992, Lensink et al 2001b, Van de Water et al 2003, Grigor et al 2004). Ceci est vraisemblablement dû à la présence des animaux dans un environnement et une situation non familiers, et aux efforts musculaires liés aux mouvements du camion. Le confinement des bovins dans le camion et le transport entraîne une augmentation de la fréquence de miction et de la cortisolémie lorsque le camion se met en mouvement.

Les réponses comportementales et physiologiques s'atténuent ensuite après environ deux heures de transport (Tarrant 1990). La fréquence cardiaque chez les veaux baisse progressivement après 30 à $60 \mathrm{mn}$ de transport (Lensink et al 2001a, Van de Water et al 2003). Les conditions du transport (température, courants d'air, conduite du chauffeur), la densité et l'emplacement des animaux dans le camion influencent le stress psychologique et les efforts physiques (Kenny et Tarrant, 1987, Van de Water et al 2003). Une durée de transport longue et une densité faible favorisent le couchage des veaux qui peuvent alors être blessés par ceux restant debout (Grigor et al 2004).

La position des veaux dans le camion peut influencer la réponse cardiaque et la cortisolémie, plus élevées chez les veaux dans la partie avant et arrière, respectivement. La différence s'explique probablement par des différences en termes de vibrations et d'efforts physiques pour maintenir l'équilibre, de changements de température et de courants d'air (Randall et al 1995, Van de Water et al 2003). Les gros bovins s'orientent préférentiellement parallèlement ou perpendiculairement au déplacement, afin de maintenir l'équilibre. Le maintien de l'équilibre dépend également de la conduite du chauffeur, de l'état des routes et de la densité (Kenny et Tarrant 1987a, 1987b). Une densité trop élevée empêche les bovins de 
s'orienter par rapport à la route et de se relever en cas de chute et augmente la cortisolémie. Une densité trop faible empêche les bovins de s'appuyer les uns sur les autres pour aider au maintien de l'équilibre. Une perte d'équilibre suivie par une chute risque de provoquer des blessures ou l'écrasement par les congénères. Le maintien de l'équilibre peut être à l'origine d'une grande fatigue : après $15 \mathrm{~h}$ de transport, des bovins adultes présentaient des augmentations significatives des taux plasmatiques de créatine kinase (Knowles 1999, Tarrant 1990). Après 2 à $4 \mathrm{~h}$ de transport les bovins adultes peuvent se coucher même si le camion est en mouvement, si les conditions sont adaptées ; ceci est toutefois rare (Broom 2003).

$\mathrm{Au}$ déchargement à l'abattoir, la plupart des phénomènes potentiellement stressants se reproduisent : les manipulations, un nouvel environnement, et pour les veaux surtout, des mélanges avec des congénères non familiers. Toutefois, les abattoirs disposent d'équipements adéquats et le déchargement se déroule donc généralement plus vite que le chargement. La fréquence cardiaque des veaux pendant le déchargement montre à peu près le même niveau que pendant le chargement à la ferme, c'est-à-dire une augmentation environ 70 à $75 \%$ par rapport au niveau dans la loge d'élevage (tableau 1, Lensink et al 2001b).

Pour les gros bovins, l'attente à la bouverie de l'abattoir peut permettre une récupération suite aux efforts et au stress du déchargement. Une attente d'au moins 24 à 48 h permet aux animaux de retrouver un état comportemental, physiologique et métabolique normal (Knowles 1999, Mounier et al 2006a). Chez le veau, le déchargement provoque des réponses physiologiques plus prononcées que l'attente à la bouverie, probablement en partie à cause de l'effort physique. Immédiatement après le transport, le cortisol est toujours élevé mais atteint un niveau proche des valeurs d'avant le transport après $2 \mathrm{~h}$ d'attente en bouverie (Lensink et al 2001a, Van de Water et al 2003, Grigor et al 2004). Néanmoins, l'environnement global autour de la bouverie (bruits, odeurs, nouveauté...) et la durée d'attente dans la bouverie peuvent influencer l'agitation des animaux. Plus l'attente est longue (dépassant 2 h), plus les veaux se chevauchent et se couchent avec les risques de glissades et de blessures (Grigor et al 2004).
Concernant la phase de conduite au poste d'abattage et l'étourdissement des bovins et des veaux, peu de données sont disponibles. Les aspects anxiogènes connus concernent la manipulation par l'Homme, l'isolement social dû à l'avancement en file indienne, l'obscurité du poste d'abattage ou encore une mauvaise ventilation ne permettant pas l'évacuation des odeurs (Grandin 2005). L'utilisation de l'aiguillon électrique pour faire avancer les bovins augmente fortement les vocalisations, signe de stress chez les bovins (Grandin 2001).

Enfin, du point de vue du stress de l'animal, l'étourdissement est préférable à l'abattage rituel sans étourdissement où la perte de conscience est beaucoup plus lente (Daly et al 1988, Bager et al 1992).

\section{4 / Poissons d'élevage}

La préparation à l'abattage nécessite le regroupement des poissons. Ils sont soit abattus à côté du bassin, soit transportés sur une courte distance jusqu'à une salle d'abattage du site d'élevage, soit transportés sur des distances plus ou moins longues jusqu'à un site d'abattage spécialisé. Le chargement nécessite d'extraire les poissons de leur milieu et souvent de les exposer à l'air. Une période de jeûne (de un à quelques jours), selon la température pour vider le contenu du tube digestif, est nécessaire pour maintenir une bonne qualité de l'eau lors du transport et pour garantir la qualité sanitaire ultérieure du produit. La méthode d'étourdissement choisie dépend de l'espèce, pour des raisons pratiques ou physiologiques. La réglementation pour les poissons stipule l'évitement de toute souffrance non nécessaire. Elle n'est pas plus précise car le bien-être des poissons dépend au moins autant des conditions (qualité de l'eau, oxygène, température) que des procédés d'abattage.

Ces étapes de pré-abattage se traduisent de manière plus ou moins marquée, selon les espèces et les conditions, par des réponses de stress : altérations hormonales et comportementales (Wendelaar Bonga 1997), ou une diminution des réserves en glycogène hépatique qui peut également être liée aux efforts des poissons lors de la pêche (Paxton et al 1984).

Les étapes de regroupement et de chargement sont les deux étapes les plus stressantes pour les poissons
(Miles et al 1974, Specker et Schreck, 1980). Le stress se traduit par une altération de l'activité natatoire (Sigismondi et Weber 1988) et des taux plasmatiques du cortisol et du glucose, entre autres (Mazeaud et al 1977). Cependant, selon les espèces, les différentes techniques de chargement utilisées (pompe, épuisette, tapis...) n'induisent pas le même niveau de stress (Wagner et Driscoll 1994).

Lorsqu'il est effectué dans de bonnes conditions de qualité d'eau, le transport peut permettre chez certaines espèces une récupération se traduisant par exemple par une baisse du cortisol plasmatique (Maule et al 1988). L'oxygène et la température doivent être proches de celles de l'élevage et le niveau de dioxyde de carbone et des déchets azotés bas, grâce à une durée de jeûne adaptée. A l'heure actuelle, il n'existe pas de normes de densité lors du transport ; la densité maximale acceptable dépend de la qualité de l'eau (des systèmes de traitement de l'eau pouvant être embarqués ou non), de la durée de transport et de l'espèce (Wedemeyer 1992). Après le déchargement, la récupération totale, indiquée par exemple par une reprise alimentaire, est plus ou moins rapide selon les espèces et peut durer plusieurs jours (Bandeen et Leatherland 1997).

Comme les autres espèces traitées dans cette revue, les poissons ont des organes sensoriels et perçoivent des stimuli douloureux (Huntingford et al 2006). L'abattage doit donc conduire à une perte de conscience et de sensibilité le plus rapidement possible (Robb and Kestin 2002). Aujourd'hui l'abattage des poissons d'élevage est réalisé par différentes méthodes induisant des niveaux de stress variables selon l'espèce et la façon dont elles sont mises en pratique. La saignée et l'asphyxie à l'air libre sont des méthodes de mort lente (de quelques minutes à quelques heures selon la température et l'espèce) et par conséquent, peu acceptables d'un point de vue du respect de l'animal (Robb et Kestin 2002).

Appliquée correctement, la percussion provoque la mort quasi instantanée. Elle peut se faire manuellement ou, plus rarement, par un système automatique. L'automatisation est difficile à optimiser et l'application manuelle nécessite précision et force, qui peut diminuer après environ $30 \mathrm{mn}$ de pratique. L'automatisation a démontré son utilité pour plusieurs espèces mais peut rendre difficile la saignée (Van de Vis et al 2003). 
L'anesthésie au $\mathrm{CO}_{2}$, obtenue en passant un flux de $\mathrm{CO}_{2}$,dans l'eau, provoque une très forte activité musculaire pendant les premières minutes puis chez le saumon la mort après $5 \mathrm{mn}$ (Robb et al 2000). Cette méthode est stressante, comme l'indiquent des changements des taux d'hématocrite et de glucose et cortisol plasmatiques, chez plusieurs espèces (Marx et al 1999).

L'électronarcose consiste en l'application d'un courant dans l'eau. En respectant un paramétrage adéquat elle rend l'anguille inconsciente (absence de réponse à un stimulus douloureux) quasi instantanément (Van de Vis 2003). Par contre, chez le turbot, l'électronarcose induit une inconscience courte, associée à une production importante de mucus, interprétée comme signe de stress (Morzel et al 2003). Chez les salmonidés, en adaptant les paramètres (voltage, durée), on peut obtenir une inconscience (Roth et al 2003).

La destruction partielle du cerveau par l'introduction d'un poinçon ou couteau (spiking) nécessite une maîtrise technique du geste mais provoque une mort très rapide chez la dorade (Nakayama et al 1996). Néanmoins si le geste est mal maîtrisé des signes de souffrance, une activité musculaire intense, ont été observés (Robb et al 2000).

La balnéation des poissons dans une eau très froide (autour de $0^{\circ} \mathrm{C}$ ) peut, chez certaines espèces, être assimilée à une anesthésie létale. Cette méthode est très utilisée pour le bar et la dorade, des poissons d'eau de mer tempérée (Huidobro et al 2001). Elle n'est pas adaptée pour le turbot ou la truite, des réactions de stress importantes ayant été notées chez ces deux espèces (Robb et al 2000, Morzel et al 2003).

La méthode d'étourdissement la plus adaptée dépend essentiellement de l'espèce car selon leur habitat, certains poissons sont très résistants au froid (balnéation dans de l'eau froide) ou à la raréfaction d' $\mathrm{O}_{2}$. Pour certaines espèces comme la dorade, l'anguille et le maquereau africain, la morphologie du crâne prévient une transmission suffisante de la concussion vers le cerveau nécessaire pour induire une perte de conscience (Van de Vis et al 2003). A cause de la précision requise, la méthode du «spiking» est réservée aux espèces de taille suffisante $(>4-5 \mathrm{~kg})$, que l'on maintient individuellement (saumon, thon). Enfin, dans un souci d'optimisation des conditions d'abattage, différentes méthodes peuvent être associées. Ainsi la méthode de «live chilling», associant une anesthésie à froid $\left(1^{\circ} \mathrm{C}\right)$, une asphyxie au $\mathrm{CO}_{2}$ à $1^{\circ} \mathrm{C}$, puis une saignée, a été développée pour les saumons. Cette méthode a des avantages en terme de stress d'abattage (Skjervold et al 2001).

\section{Conclusion}

Malgré les progrès de ces dernières années, les facteurs de stress à l'abat- tage sont multiples et dépendent de l'espèce considérée. Il est important de continuer d'accroître nos connaissances sur les causes exactes de stress, d'améliorer certains équipements (liés au chargement, à la conduite des animaux et aux conditions d'attente), la maîtrise de l'ambiance, à la ferme ou en abattoir et certains procédés (transport et attentes trop longs, mélanges d'animaux d'origines différentes, formation du personnel aux manipulations, au transport et à l'abattage, entretien du matériel de l'étourdissement), mais également de maintenir, et si possible améliorer, la maîtrise des qualités des viandes. Certaines réponses comportementales et physiologiques aux conditions de pré-abattage ont un impact sur le métabolisme ante et post mortem et par ce biais sur les qualités technologiques et/ou sensorielles des viandes des volailles (Debut et al 2003, Berri et al 2005), des porcs (Terlouw et al 2006) des veaux et bovins (Lensink et al 2000, Mounier et al 2006a) et de la chair des poissons (Morzel et Van de Vis 2003). Les pratiques favorables au bien-être et à la qualité des viandes ne vont pas toujours de pair et l'enjeu futur sera de trouver un équilibre entre ces deux objectifs, par des approches sur le terrain (à la ferme, pendant le transport et en abattoir) et en laboratoire (évaluation des conditions d'abattage sur le niveau de stress et les effets de celui-ci sur la transformation du muscle en viande).

\section{Références}

Bager F., Braggins T.J., Devine C.E., Graafhuis A.E., Mellor D.J., Tavener A., Upsdell M.P., 1992. Onset of insensibility at slaughter in calves: effects of electroplectic seizure and exsanguination on spontaneous electrocortical activity and indices of cerebral metabolism. Res. Vet. Sci., 52, 162-173.

Bandeen J., Leatherland J.F., 1997. Transportation and handling stress of white suckers raised in cages. Aquacult. Int., 5, 385396.

Beattie, V.E., Burrows M.S., Moss B.W. Weatherup R.N., 2002. The effect of food deprivation prior to slaughter on performance, behaviour and meat quality. Meat Sci., 62, 413418.

Berri C., Debut M., Santé-Lhoutellier V., Arnould C., Boutten B., Sellier N., Baéza E., Jehl N., Jégo Y., Duclos M.J., Le Bihan-Duval E., 2005. Variations in chicken breast meat quality: implications of struggle and muscle glycogen content at death. Br. Poult. Sci., 46, 572-579.
Broom D.M., 2003. Transport stress in cattle and sheep with details of physiological, ethological and other indicators. Dtsch. Tierarztl. Wochenschr., 110, 83-88.

Brown S.N., Knowles T.G., Edwards J.E. Warriss P.D., 1999. Relationship between food deprivation before transport and aggression in pigs held in lairage before slaughter. Vet. Rec., $45,630-634$.

Brown S.N., Knowles T.G., Wilkins L.J., Chadd S.A., Warriss P.D., 2005. The response of pigs to being loaded or unloaded onto commercial animal transporters using three systems. Vet. J., 170, 91-100.

Cashman P.J., Nicol C.J., Jones R.B., 1989. Effects of transportation on the tonic immobility fear reactions of broilers. Br. Poult. Sci., 30, 211 221.

Daly C.C., Gregory N.G., Wotton S.B., 1987. Captive bolt stunning of cattle: effects on brain function and role of bolt velocity. Br. Vet. J., 143, 574-580
Daly C.C., Kallweit E., Ellendorf F., 1988. Cortical function in cattle during slaughter: conventional captive bolt stunning followed by exsanguination compared with shechita slaughter. Vet. Rec., 122, 325-329.

Debut M., Berri C., Baéza E., Sellier N., Arnould C., Guémené D., Jehl N., Boutten B., Jego Y., Beaumont C., Le Bihan-Duval E., 2003. Variation of chicken technological meat quality in relation to genotype and preslaughter stress conditions. Poult. Sci., 82, 1829-1838.

Debut M., Berri C., Arnould C., Guemené D., Santé-Lhoutellier V., Sellier N., Baéza E., Jehl N., Jégo Y., Beaumont C., Le Bihan-Duval E., 2005. Behavioural and physiological responses of three chicken breeds to pre-slaughter shackling and acute heat-stress. Br. Poult. Sci., 46, 527-535.

Deiss V., Astruc T., Ferreira C., Terlouw E.M.C., 2007 Anesthésie gazeuse des porcs. I. Réponses physiologiques et comportementales à différents mélanges gazeux. Viandes et Produits Carnés 25, 71-82. 
Duncan I.J.H, Slee G.S, Kettlewell P., Berry P., Carlisle A.J., 1986. Comparison of the stressfulness of harvesting broiler chickens by machine and by hand. Br. Poult. Sci., 27, 109-114.

Fernandez X., Meunier-Salaün M.C., Mormède P., 1994. Agonistic behavior, plasma stress hormones, and metabolites in response to dyadic encounters in domestic pigs: interrelationships and effect of dominance status. Physiol. Behav., 56, 841-847.

Gentle M.J., Tilson V.L., 2000. Nociceptors in the legs of poultry: implications for potential pain in pre-slaughter shackling. Anim. Welf., 9, 227-236.

Grandin T., 2005. Maintenance of good animal welfare standards in beef slaughter plants by use of auditing programs. J. Am. Vet. Med. Assoc., 226, 370-373.

Grandin T., 2001. Cattle vocalizations are associated with handling and equipment problems at beef slaughter plants. Appl. Anim. Behav. Sci., 71, 191-201.

Gregory N.G., Bell J.C., 1987. Duration of wing flapping in chickens shackled before slaughter. Vet. Rec., 121, 567-569.

Gregory N.G., Mohan Raj A.B., Audsley A.R.S, Daly C.C., 1990. Effect of $\mathrm{CO}_{2}$ on man. Fleischwirtsch., 70, 1173-1174.

Gregory N.G., Wilkins L.J., 1989. Broken bones in domestic fowl: handling and processing damage in end-of-lay battery hens. Br. Poult. Sci., 30, 555-562.

Grigor P.N., Cockram M.S., Steele W.B., McIntyre J., Williams C.L., Leushuis I.E., Van Reenen C.G., 2004. A comparison of the welfare and meat quality of veal calves slaughtered on the farm with those subjected to transportation and lairage. Livest. Prod. Sci., 91, 219-228.

Hambrecht E., Eissen J.J., Newman D.J., Smits C.H.M., den Hartog L.A., Verstegen M.W.A., 2005. Negative effects of stress immediately before slaughter on pork quality are aggravated by suboptimal transport and lairage conditions. J. Anim. Sci., 83, 440-448.

Hemsworth P.H., Barnett J.L., Hofmeyr C., Coleman G.J., Dowling S., Boyce J., 2002. The effects of fear of humans and pre-slaughter handling on the meat quality of pigs. Aust. J Agric. Res., 53, 493-501.

Hoenderken R., Van Logtestijn J.G., Sybesma W., Spanjaard W.J.M. 1979. Carbon dioxide stunning of pigs. Fleischwirtsch, 59, 1572-1578.

Huidobro A., Mendes R., Nunes M.L., 2001. Slaughtering of gilthead seabream (Sparus aura$t a)$ in liquid ice: influence on fish quality. Eur. Fd Res. Technol., 213, 267-272.

Huntingford F., Adams C., Braithwaite V.A., Kadri S., Pottinger T.G., Sandoe P., Turnbull J., 2006. Current issues in fish welfare. J. Fish Biol., 68, 332-372.

Kannan G., Mench J.A., 1996. Influence of different handling methods and crating periods on plasma corticosterone concentrations in broilers. Br. Poult. Sci., 37, 21-31.

Kannan G., Heath J.L., Wabeck C.J., Mench J.A., 1997a. Shackling of broilers: effects on stress responses and breast meat quality. $\mathrm{Br}$. Poult. Sci., 38, 323-332.

Kannan G., Heath J.L., Wabeck C.J., Souza M.C.P., Howe J.C., Mench J.A., 1997b. Effects of crating and transport on stress and meat quality characteristics in broilers. Poult. Sci., 76, 523-529.
Kenny F. J., Tarrant P. V., 1987. The reaction of young bulls to short-haul road transport. Appl. Anim. Behav. Sci., 17, 209-227.

Kim D.H., Woo J.H., Lee C.Y., 2004. Effects of stocking density and transportation time of market pigs on their behaviour, plasma concentrations of glucose and stress-associated enzymes and carcass quality. Asian-Aust. J. Anim. Sci., 17, 116-121.

Knierim U., Gocke A., 2003. Effect of catching broilers by hand or machine on rates of injuries and dead-on-arrivals. Anim. Welf., 12, $63-73$.

Knowles T.G., 1999. A review of the road transport of cattle. Vet. Rec., 144, 197-201.

Kotula K.L., Wang Y., 1994. Characterization of broiler meat quality factors as influenced by feed withdrawal time. J. Appl. Poult. Res., 3, 103-110.

Lambooij E., Gerritzen M.A., Engel B., Hillebrand S.J.W., Lankhaar J., Pieterse C., 1999. Behavioural responses during exposure of broiler chickens to different gaz mixtures. Appl. Anim. Behav. Sci., 62, 255-265.

Lambooij E., Spanjaard W., 1982. Electrical stunning of veal calves. Meat Sci., 6, 15-25.

Lensink J., Fernandez X., Boivin X., Pradel P., Le Neindre P., Veissier I., 2000. The impact of gentle contacts on ease of handling, welfare, and growth of calves and quality of veal meat. J Anim. Sci., 78, 1219-1226.

Lensink B.J., Raussi S., Boivin X., Pyykkönen M., Veissier I., 2001a. Calves' reactions to handling depend on housing conditions and previous experience with humans. Appl. Anim. Behav. Sci., 70, 187-199.

Lensink J., Fernandez X., Cozzi G., Florand L., Veissier I., 2001b. The influence of farmers' behavior towards calves on animals' responses to transport and quality of veal meat. J. Anim. Sci., $79,642-652$

Martoft L., Lomholt L., Kolthoff C., Rodriguez B.E., Jensen E.W., Jorgensen P.F., Pedersen H.D., Forslid A., 2002. Effects of $\mathrm{CO}_{2}$ anaesthesia on central nervous system activity in swine. Lab. Anim., 36, 115-126.

Marx H., Sengmuller-Sieber T., Hoffmann R., Stolle A., 1999. Stress and product quality of trout, catfish and flounder at stunning and slaughtering. Arch. Lebensmittelhyg., 50, 37-40.

Maule A.G., Schreck C.B., Bradford S. Barton B.A., 1988. Physiological effects of collecting and transporting emigrating juvenile chinook salmon past dams on the Columbia river. Trans. Am. Fish. Soc., 117, 245-261.

Mazeaud M.M., Mazeaud F., Donaldson E.M., 1977. Primary and secondary effects of stress in fish: some new data with a general review. Trans. Am. Fish. Soc., 106, 201-212.

Miles H.M., Loehner S.M., Michaud D.T., Salivar S.L., 1974. Physiological responses of hatchery reared muskellunge (Esox masquinongy) to handling. Trans. Am. Fish. Soc., 103, 336-342.

Mitchell M.A., Kettlewell P.J., Maxwell M. H., 1992. Indicators of physiological stress in broiler chickens during road transportation. Anim. Welf., 1, 91-103.

Morzel M., Sohier D., Van de Vis H., 2003. Evaluation of slaughtering methods for turbot with respect to animal welfare and flesh quality. J. Sci. Fd Agric., 83, 19-28.
Morzel M., van de Vis H., 2003. Effect of the slaughter method on the quality of raw and smoked eels (Anguilla anguilla L.). Aquacult. Res., $34,1-11$.

Mounier L., Dubroeucq H., Andanson S. Veissier I., 2006a. Variations of meat $\mathrm{pH}$ of beef bulls in relation to conditions for transfer to slaughter and previous history of animals. J. Anim. Sci., 84, 1567-1576.

Mounier L., Veissier I., Andanson S., Delval E., Boissy A., 2006b. Mixing at the beginning of fattening moderates social buffering in beef bulls. Appl. Anim. Behav. Sci., 96, 185-200.

Mounier L., Marie M., Lensink B.J., 2007. Facteurs déterminants du bien-être des ruminants en élevage. INRA Prod. Anim., 20, 65-72.

Nakayama T., Toyoda T, Ooi A., 1996. Delay in rigor mortis of red sea-bream by spinal cord destruction. Fish. Sci., 62, 478-482.

Paxton R., Gist D.H., Umminger B.L., 1984. Serum cortisol levels in thermally-acclimated goldfish (Carassius auratus) and killifish (Fundulus heteroclitus): implications in control of hepatic glycogen metabolism. Comp. Biochem. Physiol., 78B, 813-816.

Pérez M.P., Palacio J., Santolaria M.P., Acena M.C., Chacon G., Verde M.T., Calvo J.H., Zaragoza M.P., Gascon M., Garcia-Belenguer S., 2002. Influence of lairage time on some welfare and meat quality parameters in pigs. Vet. Res., $33,239-250$.

Raj A.B.M., 2004. Stunning and slaughter of poultry. In: Poultry meat processing and quality, Mead G.C. (Ed), CRC-Woodhead Publishing Ltd, Cambridge, UK, 2, 21-37.

Randall J.M., Stiles M.A., Geers R., Schütte A., Christensen L., Bradshaw R.H., 1995. Vibration on pig transporters: implications for reducing stress. Proc. EU Seminar: new information on welfare and meat quality of pigs as related to handling, transport and lairage conditions, Mariensee, Allemagne, 29-30 juin, 143-159.

Robb D.H.F., Kestin S.C., 2002. Methods used to kill fish: Field observations and literature reviewed. Anim Welf., 11, 269-282.

Robb D.H.F, Wotton S.B., Mckinstry J.L., Sorensen N.K., Kestin S.C., 2000. Commercial slaughter methods used on Atlantis salmon: determination of the onset of brain failure by electroencephalography. Vet. Rec., 147, 298303.

Roth B., Imsland A., Moeller D., Slinde E., 2003. Effect of electric field strength and current duration on stunning and injuries in market-sized Atlantic salmon held in seawater. North Am. J. Aquac., 65, 8-13.

Tarrant P.V.,1990. Transportation of cattle by road. Appl. Anim. Behav. Sci., 28, 153-170.

Sartorelli P. Dominoni S., Agnes F., 1992. Influence of duration of simulated transport on plasma stress markers in the calf. J. Vet. Med., 39,401 .

Sigismondi L.A., Weber L.J., 1988. Changes in avoidance response time of juvenile chinook salmon exposed to multiple acute handling stresses. Trans. Am. Fish. Soc., 117, 196-201.

Skjervold P.O., Fjaera S.O., Ostby P.B., Einen O., 2001. Live-chilling and crowding stress before slaughter of Atlantic salmon (Salmo salar). Aquaculture, 192, 265-280.

Specker J.L., Schreck C.B., 1980. Stress responses to transportation and fitness for marine survival in coho salmon (Oncorhynchus 
kisutch) smolts. Can. J. Fish. Aquatic Sci., 37, 765-769.

Terlouw E.M.C., Rybarczyk P., 2007. Explaining and predicting differences in meat quality through stress reactions at slaughter: the case of Large White and Duroc pigs. Soumis à Meat Sci.

Terlouw E.M.C., Astruc T., Berne A., Haye E., Laville E., Lhoutellier-Santé V., Monin G. Morzel M., Sayd T., Hamelin M., Vernin P., 2006. Elevage de porcs extérieurs. Effets de la génétique et des conditions d'abattage sur le comportement, la physiologie et les qualités des viandes. Viandes et Produits Carnés, 25, 43-53.

Van de Vis H., Kestin S., Robb D., Oehlenschlager J., Lambooij B., Münkner W., Kuhlmann Kloosterboer K., Tejada M., Huidobro A., Ottera H., Roth B., Sorensen N.K.,
Akse L., Byrne H., Nesvadba P., 2003. Is humane slaughter of fish possible for industry? Aquacult. Res., 34(3), 211-220.

Van de Water G., Verjans F., Geers R., 2003. The effect of short distance transport under commercial conditions on the physiology of slaughter calves; $\mathrm{pH}$ and colour profiles of veal. Livest Prod. Sci., 82, 171-179.

Veissier I., Beaumont C., Lévy F., 2007. Les recherches sur le bien-être animal : buts, méthodologie et finalité. INRA Prod. Anim., 20, 3-10.

Wagner E.J., Dirscoll D.M., 1994. Physiological stress responses of cutthroat trout to loading by fish pump, conveyor or dip net. J. Appl. Aquacult., 4, 19-27.

Warriss P.D., Bevis E.A., Brown S.N., Edwards J.E., 1992. Longer journeys to proces- sing plants are associated with higher mortality in broiler chickens. Br. Poult. Sci., 33, 201-206.

Warriss P.D., Wilkins L.J., Knowles T.G., 1999. The influence of ante-mortem handling on poultry meat. In: Poultry Meat Science, Richardson R.I., Mead G.C. (Eds), Oxon, CABI Publishing, 217-230.

Wedemeyer G. A., 1992. Transporting and handling smolts. Wld Aquac., 23, 47-50.

Wendelaar Bonga S. E., 1997. The stress response in fish. Physiol. Rev., 77, 591-625.

Wotton S.B., O'Callaghan M., 2002. Electrical stunning of pigs: the effect of applied voltage on impedance to current flow and the operation of a fail-safe device. Meat Sci., 60, 203-208.

\section{Résumé}

Malgré les progrès de ces dernières années, la période de pré-abattage est une source de stress pour les animaux. Les procédés d'abattage nécessitent des regroupements et des mélanges d'animaux, l'enlèvement du milieu habituel et l'introduction dans des environnements non familiers, le transport, la manipulation par l'homme et le jeûne, et entraînent parfois des mauvaises conditions d'ambiance. Ils sont souvent générateurs de stress d'origine physique (fatigue, faim, douleur, inconfort physique) et psychologique (peur, stress social). Les techniques d'étourdissement sont parfois mal maîtrisées ou mal adaptées. Ces procédés entraînent des réponses comportementales, physiologiques et métaboliques qui sont utilisées pour évaluer le niveau de stress de l'animal. Afin de mieux respecter le bien-être animal à l'abattage, il est nécessaire d'élargir nos connaissances des causes de stress. L'effet négatif que peuvent avoir ces réponses sur les qualités des viandes est succinctement abordé.

\section{Abstract}

\section{Effect of pre-slaughter conditions on stress and well-being of farm animals}

Despite progress made in recent years, the pre-slaughter period remains stressful for animals. Current slaughter procedures involve regrouping and mixing animals, removing them from their familiar environment and introducing them into unfamiliar settings, transporting, handling, and depriving them of food, and are sometimes associated with poor quality of surroundings. These often cause stress, which can be both physical (fatigue, hunger, pain, discomfort) and psychological (fear, social stress). Stunning techniques are sometimes poorly controlled or unsuitable. Slaughter procedures cause behavioural, physiological and metabolic responses that are used to determine the animal's stress levels. To improve animal welfare, further knowledge of the exact causes of stress is required. The possible negative effects of stress responses on meat quality are also briefly addressed.

TERLOUW E.M.C., ARNOULD C., AUPERIN B., BERRI C., LE BIHAN-DUVAL E., LEFEVRE F., LENSINK J., MOUNIER L., 2007. Impact des conditions de pré-abattage sur le stress et le bien-être des animaux d'élevage. INRA Prod. Anim., 20, 93-100. 